\title{
Regulator synthesis for the self-sensing control system of the proportional electromagnet dc based on reduced-order models
}

\author{
Danil Shaykhutdinov ${ }^{1, *}$, Nikolay Gorbatenko ${ }^{1}$, Yuri Manackov ${ }^{1}$, and Konstantin Shirokov ${ }^{1}$ \\ ${ }^{1}$ SRSPU (NPI), Department of Information and Measuring Systems and Technologies, 346428 Novocherkassk, Russia
}

\begin{abstract}
Modern DC proportional solenoid control systems use current values in magnetizing coils, or specialized sensors for position determination. These methods do not provide the possibility of accurate control and diagnostics, in case of aiming to the miniaturization of finished devices. In this article, it is proposed to use methods of self-sensory identification of the moving element position based on the method of the full-scale-model experiment. The functioning of the method is based on the electromagnet model obtained by the reduced-order model approach. These models have an advantage in the calculation speed in comparison with finite element models and have an advantage in accuracy in comparison with analytical models. Ansys Electronics is used to obtain the model. The electromagnetic control system is proposed. Its model is implemented in the system Matlab Simulink. Synthesis of PID-regulator parameters using Matlab is performed. The results of a study of a control system for a given displacement with the aid of the obtained control system are presented.
\end{abstract}

\section{Introduction}

An important problem of modern science is the provision of high reliability of industrial and household technical systems based on electromagnetic mechanisms, for example $[1,2]$. This is one of the main conditions for preventing system accidents and catastrophes. Existing methods and tools of monitoring, control and diagnosis these systems do not use achievements of modern information technologies based on modelling and methods of data mining. Integration of diagnostic functions into the device control system can significantly improve accuracy and reliability. The solution of this problem requires the development of the foundations for the functioning of systems for the diagnosis of electromagnetic mechanisms. Solving the problem of creating theoretical foundations and modern intelligent diagnostic mechanisms will help lay the foundation for the further development of intelligent systems in the field of smart factoring $[3,4]$, so relevant at the present time $[5,6]$.

To solve this problem, as the first step, it is proposed to develop new control systems with diagnostics functions based on reduced-order mathematical models for assessing the efficiency of device operation.

Purpose of work is to increase the accuracy and efficiency of the operation of electrical systems, improve the reliability of the devices operating in facilities with increased responsibility and those industries where electrical products operate under difficult operating conditions.
Electromagnets, as a transformers, are the most common representatives of electromagnetic structures. Proportional electromagnets are the most complex system. This type of electromagnetic drives requires systems that can control the parameters of the movement of the electromagnets of the elements. In the development of new products, it is important to adjust the regulators and make a comprehensive testing of electromagnets [7, 8]. In addition, high reliability requirements are designated for this class of devices when it is used as part of increasing the responsibility of systems. The complexity and continuous improvement of magnetic systems currently require a new highprecision approach to controlling the position of moving elements [9]. Automated systems controlling the production of intelligent electric drives are necessary to implement an effective approach. One of the main elements of production systems are the stands for diagnostics and adjusting the parameters of the regulator. When developing the experimental production of new proportional DC electromagnets, this stand can be presented in the form of an automated system for research and complex tests. These operations are designed to improve the performance of processes for optimizing the electromagnets of structures, including using new materials [10] or materials known in limiting regimes [11].

In case of a high complexity of the control object (for example, a current source having a limit of power or configuration of the electromagnetic system of an

\footnotetext{
* Corresponding author: d.v.shaykhutdinov@gmail.com
} 
electromagnet has a high geometric complexity) or in the absence of complete a priori information about the object, it becomes necessary to apply intelligent control technologies [12, 13]. Currently, a very wide range of publications on intelligent technologies, which confirms the relevance and popularity of these control systems.

\section{Materials and Methods}

\subsection{Self-sensing control method}

The proposed approach is based on the following assumptions. The position of the electromagnet movable element $x$ defines a degree of magnetic circuit closure $d$. The degree of of magnetic circuit closure $d$ determines the level of magnetic flux $F$ at a preset value of magnetopropellent $I w$. The level of a magnetic flux has direct interrelation with the flux linkage of the winding of magnetic conductor $\psi$. Thus, there is physical interrelation between the weber-ampere characteristic of electromagnet $\psi(I w)$ and the position of its movable element, $\quad x: \quad x \rightarrow d \rightarrow F(I w) \rightarrow \psi(I w)$. Based on this assumptions, the automatic control system (Fig. 1) is proposed.

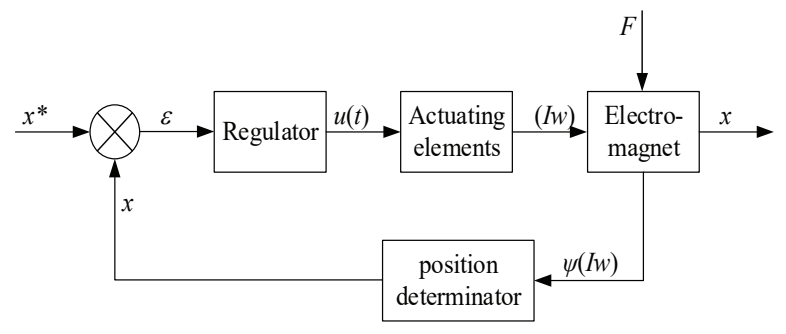

Fig. 1. Automatic control system

The system (Fig. 1) consists of: Regulator - the element that forms the control actions, Actuating elements - the aggregate of the current source and the magnetizing coil of the electromagnet, Elecromagnet - the proportional electromagnet of direct current, position determinator the element for determining the position of the movable element of the electromagnet. For the formation of the latter, the application of the method of full-scale-model tests is proposed.

\subsection{Full-scale-model tests method}

Full-scale-model tests method refers to a group of iterative methods for solving inverse problems. Its peculiarity is that the results of the experiment are used both as input data for modeling the field of the system, and as a criterion for estimating the accuracy of the numerical solution of this problem. In general, the iterative process is implemented as a change in the configurable parameter of the model by summing the value of the given parameter obtained in the previous step and some correction value. At that, to determine the value of the parameter to be adjusted at the zero iteration, the value of the results of the physical experiment is used. The correction value is found as a function of the difference between the result of the physical experiment for measuring the initial parameter and the result of the model experiment for determining the same parameter when the value of the parameter being tuned into the model is substituted into the model. The iterative process is completed, if the necessary accuracy of the numerical solution of the identification problem is reached, defined as the difference between the result of the physical experiment in measuring the initial parameter and the result of the model experiment for determining the same parameter (Fig. 2).

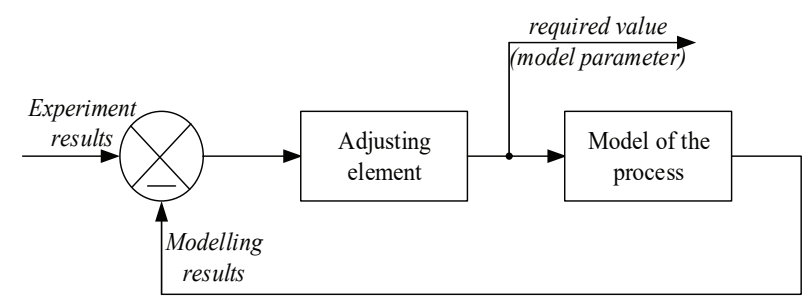

Fig. 2. Realization of measurement based on full-scale-model tests method

We proposed the method for obtaining the measurement information about the state of the technical objects being studied, which differs from the known ones in that the operation for calculating the results of measurements is replaced by the operation of solving the inverse problem of identifying the model of the system under investigation with the subsequent determination of the sought value from the simulation results [14]. In the case under consideration, the initial data (experimental results) and simulation results will be the weber-ampere characteristic values. As the output data - the position of the moving element of the electromagnet. To calculate these parameters, in the first case it is necessary to have a mathematical description of the control object.

\subsection{Electromagnetic field mathematical modelling method}

The methods of modeling magnetic fields on the basis of the equations of magnetic circuits, analytical methods for calculating the field and the equations of the electromagnetic field are considered. For carrying out of experimental researches the method of mathematical modeling of an electromagnetic field of objects on the basis of system of Maxwell's equations is chosen. In developing the mathematical model, the following assumptions were made: as a result of the investigation of magnetic DC systems or industrial frequency current, it is assumed that there are no free charges in the system; the field is assumed to be quasistationary, which implies the absence of a delay in the electromagnetic wave in the dielectric; the losses due to hysteresis when the ferromagnetic metals are heated are not taken into account because of their insignificance in comparison with the eddy current losses [15]. The magnetic field equation has the form of 


$$
\operatorname{rot} \vec{H}=\vec{\delta} ; \operatorname{div} \vec{B}=0,
$$

wherein $\vec{B}, \vec{H}$ - vectors of the magnetic flux density and magnetic field strength, respectively, $\vec{\delta}$ - the known current density in the excitation winding $V_{\mathrm{obm}}$.

For the system (1) $\vec{B}=\mu_{0} \vec{H}$ - in the air $V_{0}$ and in the excitation winding $V_{\mathrm{obm}} ; \vec{B}=\mu_{F e} \vec{H}-$ in ferromagnets $V_{F e} ; B_{n}^{+}=B_{n}^{-} ; H_{\tau}^{+}=H_{\tau}^{-}$.

On the basis of the equation $\operatorname{div} \vec{B}=0$, we introduce the magnetic vector potential $\vec{A}$ by relations $\operatorname{rot} \vec{A}=\vec{B}$; $\operatorname{div} \vec{A}=0$. Using $\vec{A}$, we transform the system (1). As a result, we obtain

$$
\begin{gathered}
\operatorname{div}\left(\frac{1}{\mu_{0}} \operatorname{grad} \vec{A}\right)=\left\{\begin{array}{l}
\vec{\delta}-\text { in } V_{\text {obm }} ; \\
0-\text { in } V_{0} ;
\end{array}\right. \\
\operatorname{div}\left(\frac{1}{\mu} \operatorname{grad} \vec{A}\right)=0-\text { in } V_{F e} .
\end{gathered}
$$

At the interface we have

$$
A^{+}=A^{-} ; \frac{1}{\mu^{+}} \frac{\partial A^{+}}{\partial n}=\frac{1}{\mu^{-}} \frac{\partial A^{-}}{\partial n} .
$$

The solution of system (2) with condition (3) can be conveniently found by using the method of finite differences, the method of boundary elements, the variational version of the finite element method (FEM) [6]. The main criterion in the analysis, due to the complexity of the objects under study, is the possibility of solving nonlinear problems for bodies of complex shape. A finite element method was chosen that provides ample opportunities for describing the complex geometry of the objects under study, taking into account existing nonlinearities in the analysis of field problems, constructing calculation algorithms and automating the process of processing simulation results in modern software complexes. Minimization of the functional corresponding to problem (2), (3) leads to a system of algebraic equations.

$$
\sum_{k} \frac{1}{\mu^{k}} \sum_{j} \beta_{i j}^{k} A_{i}=\frac{1}{3} \sum_{k} \mu_{0} \delta^{k} S^{k}, k=1,2, \ldots, N,(4)
$$

where $\beta_{i j}^{k}=\iint_{V^{k}} \operatorname{grad} \psi_{i}^{k} \operatorname{grad} \psi_{j}^{k} d V, \psi_{i}^{k}$ and $\psi_{j}^{k}$ - the

basic functions of the $k$-th finite element; $S^{k}$ - area of the face of the $k$-th finite element.

But solving the system (4) requires experimentally large amounts of time and computing resources. Thus, this approach can't be directly applied in problems of high-speed systems control, for example proportional electromagnets DC. To solve this problem the using of reduced-order models approach is proposed.

\subsection{Reduced-order models}

The problem of reducing the number of variables of the finite element model to a number of main components can be solved using the reduced-order model (ROM) approach [16-22]. This approach allows us to take into account only the most important features of the modeling object with the help of several state variables and several ordinary differential equations. As a result of applying this approach, the original matrix of the system (4) is transformed into a simplified one. A simplified matrix can be more effectively used to solve real-time control problems. The obtained results can be integrated into the MatLab Simulink system, for example, to automate the synthesis of the control system regulator. In general view, a decrease in the order of the FEM model can be described as the transformation of some initial system:

$$
\left\{\begin{array}{l}
\dot{z}(t)=A z(t)+B g(t) \\
y(t)=C z(t)
\end{array}\right.
$$

wherein $z$ - the state vector, $f$ - the input vector, $y$ - the output vector, to the system of equations of:

$$
\left\{\begin{array}{l}
\dot{z}_{\text {rom }}(t)=A_{\text {rom }} z_{\text {rom }}(t)+B_{\text {rom }} g(t) \\
y(t)=C_{\text {rom }} z_{\text {rom }}(t)
\end{array}\right.
$$

It should be noted, that the dimension of the vectors of input actions and output values, for system (5) is preserved. To obtain ROM from the full FEM models, various methods are used, which are described in detail in [22]. In particular, the ANSYS Electronics system, which performs finite element modeling of objects [15], allows to obtain ROM using the built-in algorithm based on modal decomposition.

By applying the approach of reduced-order models and solving the result system, we find $B_{i}^{0}(i=1,2, \ldots, N)$, where $N-$ a number of measuring transducers. Then, we will following the next calculations sequence:

1. Calculate the square of the norm of the difference $J_{i}=\left\|B_{i}^{0}-B_{i}^{*}\right\|^{2}$.

2. Check the condition $J_{i} \leq \varepsilon$, where $\varepsilon$-accuracy of determining the minimum of the functional $J_{i}$.

3. If the condition of point 4 is satisfied, the solution is obtained and it equals $\vec{f}^{0}$. Otherwise, using the gradient method of minimizing the functional $J$, we find the next approximation $\vec{f}^{1}$. Here the ratio $\vec{f}^{k+1}=\vec{f}^{k}-[\alpha] \operatorname{grad} J^{k}$ is used, wherein $[\alpha]-$ a diagonal matrix of steps.

4. Return to point 3 , using the new $\vec{f}$ values.

\section{Results and discussion}

\subsection{Experiment description}

To realize a system for the study of proportional direct current magnets, the National Instruments platform was chosen. The industrial computer is based on the NI PXIe1078 chassis under the control of the NI PXI-8133 controller, with the addition of the NI PXIe-6341 multifunctional device, NI PXIe-4071 multimeter, NI 
PXI-4130 power supply. To control the operation of the electromagnet, a dedicated NI sbRIO-9636 board is used, operating on the basis of FPGA and a real-time controller. To determine the reference values of the position of the moving element, a distance sensor of type LS5-15 / 10232-2-V- (12-24) -A is used. The object of research is an electromagnet of the KTS type P25A00-24.

To implement the software, the NI LabVIEW programming environment is used. To implement the model-based tests, Ansys Electronics software package was used, which uses the finite element method to calculate the magnetic fields. In Fig. 3 shows the appearance of the electromagnet under investigation and its model.

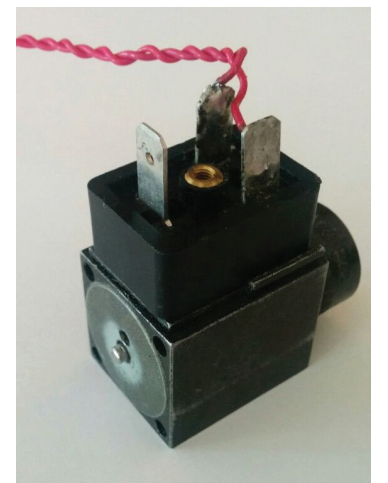

a

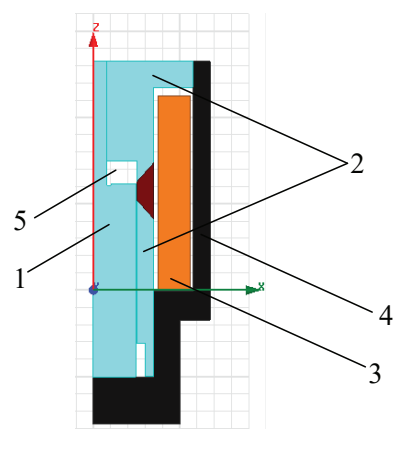

b
Fig. 3. Proportional electromagnet DC: $a-$ real look, $b$ - twodimensional axisymmetric model: 1 - anchor, 2 - magnetic core, 3 - magnetizing coil, 4 - housing, 5 - air gap

The experiment was carried out as follows. After connecting the electromagnet's electrical contacts to the system for research, the NI PXIe-4071 measures the electrical parameters of its windings. At the second stage, a pulse signal is sent to the working winding of the electromagnet (using the sbRIO-9636 FPGA, by means of a controlled current source) sufficient to make the complete armature stroke, senseless measurement of its weber-ampere characteristic (without setting additional measuring winding - and voltage on the working winding by means of the NI PXIe-6341 ADC with subsequent calculation of the weber-ampere characteristic in NI PXI-8133) and fixation of the anchor position versus time (using LS5 and NI PXI-8133). At the next stage, the magnetic parameters of the electromagnet model are tuned according to the results of the method of the full-scale-model experiment.

\subsection{Experiment results}

As a result of the physical experiment, the dependence of the electromagnet movable element displacement $x$ at the time $t$ was obtained (Fig. 4). This dependence is obtained under the condition that there is no purposeful regulation of the current values in the magnetizing coils of the electromagnet.

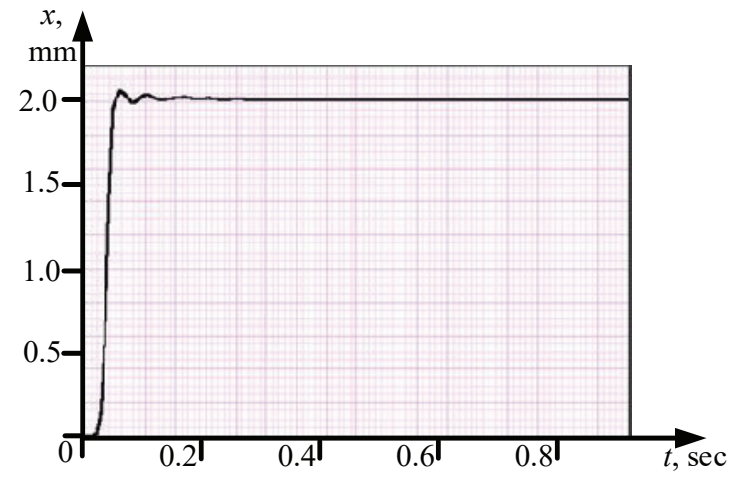

Fig. 4. The variation of the movable element position. The electromagnet operating cycle

As a result of the physical experiment, a weberampere characteristic of the electromagnet operating cycle was also obtained. The Weber-ampere characteristic of the electromagnet operating cycle was subjected to a study using the full-scale-model test method described above. An example of the result of applying the method of full-scale-model tests to obtain a model adequate to the results of measuring the weberampere characteristic of the electromagnet operating cycle is shown in Fig. 5.

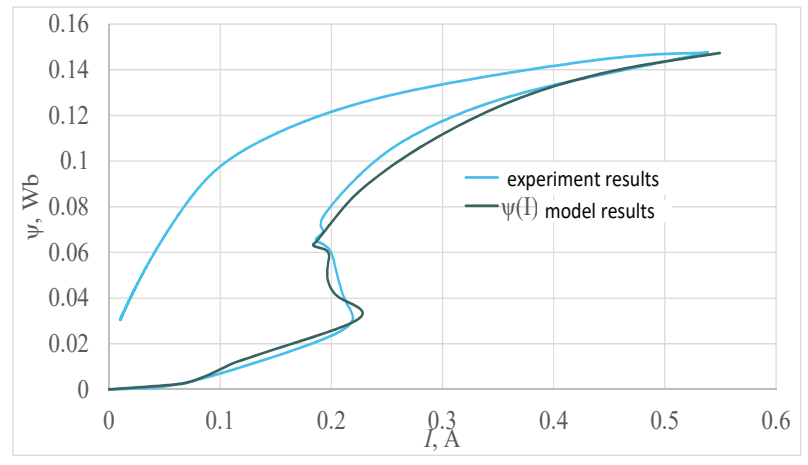

Fig. 5. The model adjustment using the method of full-scale-model tests. Results example

As it seen in Fig. 4 the final phase of the motion has an oscillatory character. This point is dangerous, as it reduces the duration of equipment operation. Where is a requirement to reduce the oscillation.

On the basis of the obtained mathematical model of an electromagnet, a regulator of a control system of a proportional DC electromagnet is synthesized. To configure its parameters, it is suggested to use the Matlab package. The controller is selected as the type PID controller. To solve this problem, the following actions must be performed. The variables $K p, K i, K d$ are initialized in the Matlab command window according to the Ziegler-Nichols method. Next, the parameters of the PID Controller block are set by entering the $K p$ variable in the Proportional parameter field, in the Integral $-K i$ field, and in the Derivative $-K d$ field. The restrictions imposed on the output of the system (the Transfer Fcn block) are specified. A corridor is installed, within which the input signal of the NCD Outport must be located. Next, automatic adjustment of the control parameters is 
performed by optimization. At the end of the optimization process, the resulting values of the custom variables are used in Matlab Simulink.

The result of modeling the moving element displacement is shown in Fig. 6.

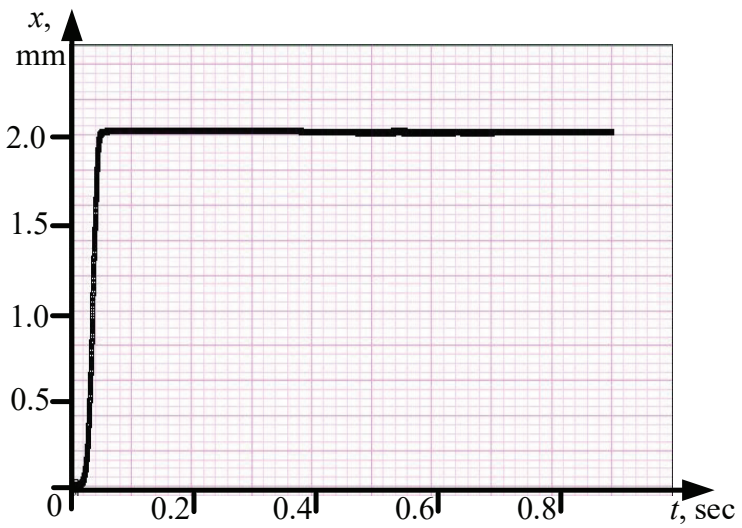

Fig. 6. Modeling the moving element displacement. Results example

\section{Conclusion}

The problem of self-sensing control of proportional DC electromagnets is considered. Self-sensor principle allows to exclude control basing on the device sensors, without additional sensors. To determine the position of the moving element, the weber-ampere characteristic of the electromagnet operating cycle is used. The relationship between the position of the moving element and the weber-ampere characteristic is established using the method of full-scale-model tests. To implement this method, it is proposed to use the finite element method. The corresponding system of equations undergoes a transformation using the approach of reduced-order models. This approach allows us to reduce the time spent on solving the system of equations and increase the efficiency of controlling the electromagnet.

This article is based on results of the project No. SP 4108.2015.1, implemented under the program "Scholarship provided by the President of the Russian Federation for young scientists and graduate students engaged in advanced research and development in priority areas of modernization of the Russian economy" using equipment of shared facility "Diagnosis and energy-efficient electrical equipment" (NPI).

\section{References}

1. V. I. Dubrov, D. V. Shaykhutdinov; K. M. Shirokov, S.V. Akhmedov, N. I. Gorbatenko, 2015 International Siberian Conference on Control and Communications, SIBCON 2015, 7147317 (2015).

2. V. I. Dubrov, D. V. Shaykhutdinov, K. M. Shirokov, Pro. Eng., 129, 184-190 (2015).

3. D. Shaykhutdinov, R. Leukhin, I. Stensenko, 2016 2nd international conference on industrial engineering, applications and manufacturing (ICIEAM), 7910945 (2016).
4. D.V. Shaykhutdinov, N.I. Gorbatenko, S.V. Akhmedov, R.I. Leukhin, 2015 International Siberian Conference on Control and Communications, SIBCON 2015, 7147329 (2015).

5. V. Dubrov, R. Oganyan, D. Shaykhutdinov, 2016 2nd international conference on industrial engineering, applications and manufacturing (ICIEAM), 7910999 (2016).

6. R.G. Oganyan, N.D. Narakidze, D.V. Shaykhutdinov, N.I. Gorbatenko, S.O. Maksuta, International conference on mechanical engineering, automation and control systems 2016, 012083 (2017).

7. R.I. Leukhin, D.V. Shaykhutdinov, V.I. Leukhin, Pro. Eng., 150, 347-353 (2016).

8. D.V. Shaykhutdinov, R.I. Leukhin, K.M. Shirokov, Pro. Eng., 129, 749-753 (2015).

9. D.V. Shaykhutdinov, N.I. Gorbatenko, N.D. Narakidze, A.S. Vlasov, I. A.Stetsenko, International conference on mechanical engineering, automation and control systems 2016, 012091 (2017).

10. N.I. Gorbatenko, V.V. Grechikhin, D.V. Shaikhutdinov, Metal Sci \& Heat Treatment, 56 (1112), 609-613 (2015).

11. D.V. Shaikhutdinov, N.I. Gorbatenko, K.M. Shirokov, Metal Sci \& Heat Treatment, 56 (11-12), 618-620 (2015).

12. R.I. Leukhin, D.V. Shaykhutdinov, K.M. Shirokov, N.D. Narakidze, A.S. Vlasov, International conference on mechanical engineering, automation and control systems 2016, 012008 (2017).

13. D. Shaykhutdinov, N. Gorbatenko, R. Leukhin, R J Appl. Sci., 10 (12), 845-847 (2015).

14. N.D. Narakidze, D.V. Shaykhutdinov, K.M. Shirokov, N.I. Gorbatenko, S.G. Yanvarev, International conference on mechanical engineering, automation and control systems 2016, 012112 (2017).

15. A. Bulgakov, D. Shaykhutdinov, N. Gorbatenko, S. Akhmedov, Pro. Eng., 123, 94-100 (2015).

16. X. Xie, D. Wells, Z. Wang, T. Iliescu, J Comp. \& Appl. Math., 328, 12-29 (2018).

17. P. Bhopale, P. Bajaria, N. Singh, F. Kazi, Adv. Intell. Sys. \& Comp., 629, 60-71 (2018).

18. P.S. Reddy, K.Y. Rani, S.C. Patwardhan, Comp. \& Chem. Eng., 106, 40-56 (2017).

19. Z. Deng, H. Deng, L. Yang, Y. Cai, X. Zhao, Energy, 138, 509-519 (2017).

20. F. Massa, I. Turpin, T. Tison, Mech. Sys. \& Sign. Proc., 96, 291-302 (2017).

21. Y. Zhang, A. Combescure, A. Gravouil, Int J Num. Methods Eng., 111(2), 176-200 (2017).

22. A. Buscarino, L. Fortuna, M. Frasca, M.G. Xibilia, Int. J Control, 90(7), 1437-1445 (2017). 